\title{
Avaliação do potencial de produção de biogás por meio do lixo doméstico em edifícios residenciais 1
}

\section{Evaluation of biogas production potential by domestic garbage in residential buildings}

\section{Schmitt Schuster, Bruna 1; Machado Márquez Zampiva, Marcelo2; Taboni Junior, Luiz Roberto3; Fernandes Viotto, Hugo Gabriel4; De Angelis Neto, Generoso5; Cruz Siqueira, Jair Antonio6.}

\author{
1 Universidade Estadual de Maringá, Avenida Colombo, 5790, Brasil, \\ brunaschuster@hotmail.com; \\ 2 Universidade Estadual do Oeste do Paraná, Rua Universitária, 1619, Brasil, \\ marcelozampiva@hotmail.com; \\ 3 Universidade Estadual de Maringá, junior_Irt@hotmail.com; \\ 4 Universidade Estadual de Maringá, enghugo.orbe@gmail.com; \\ 5 Universidade Estadual de Maringá, ganeto@vem.br; \\ 6 Universidade Estadual do Oeste do Paraná, jair.siqueira@unioeste.br.
}

\begin{abstract}
RESUMO
A relação entre a gestão de resíduos sólidos e a urbanização são assuntos que não podem ser negligenciados, principalmente quando se refere à disposição final dos Resíduos Sólidos Urbanos (RSU). A Política Nacional de Resíduos Sólidos (PNRS) visa a gestão eficiente desses, incentivando o tratamento e o aproveitamento energético dos resíduos. O objetivo desta pesquisa foi avaliar o potencial de produção de biogás por meio da digestão anaeróbia dos resíduos orgânicos provenientes de edifícios residenciais situados no município de CascavelPR mediante o método de composição química de Buswell, descrito por Chernicharo (1997). Por meio de fatores de equivalência, o biogás foi convertido à GLP e energia elétrica, a fim de evidenciar e explorar seu potencial energético. De acordo com as informações obtidas, conclui-se que a geração de resíduos orgânicos na maioria dos edifícios estudados fora maior do que a média nacional. Desse modo, deve-se considerar a utilização da digestão anaeróbia como tratamento dos resíduos orgânicos, visto que esta contribui para a redução do volume de resíduos orgânicos dispostos nos aterros. Deste modo, explorando seu potencial energético a fim de contribuir para a diversificação da matriz energética nacional.
\end{abstract}

Palavras-chave: Biogás, Resíduo sólido urbano, Energia renovável.

1 SCHMITT SCHUSTER, Bruna; MACHADO MARQUEZ ZAMPIVA, Marcelo; TABONI JUNIOR, Luiz Roberto; FERNANDES VIOTTO, Hugo Gabriel; DE ANGELIS NETO, Generoso; CRUZ SIQUEIRA, Jair Antonio. Avaliação do potencial de produção de Biogás por meio do lixo doméstico em edifícios residências. In: II SIMPÓSIO NACIONAL DE GESTÃo E ENGENHARIA URBANA: SINGEURB, 2019, São Paulo. Anais... Porto Alegre: ANTAC, 2019. 


\begin{abstract}
The relation between solid waste management and urbanization are matters that cannot be neglected, especially when referring to the final disposal of Urban Solid Waste (USW). The National Solid Waste Policy (NSWP) aims at the efficient management of waste, encouraging the treatment and energetic use of such residues. This article aims to evaluate the potential of biogas production through anaerobic digestion of organic residues from residential buildings located in the city of Cascavel, PR using the method of the chemical composition of Buswell, described by Chernicharo (1997). By means of equivalence factors, biogas was converted to LPG and electrical energy in order to highlight and exploit its energy potential. According to the information obtained, it is concluded that the generation of organic waste in most of the studied buildings was larger than the national average. Therefore, the use of anaerobic digestion as a treatment of organic waste should be considered, since this contributes to the reduction of the volume of organic waste disposed of in landfills. Therefore, exploring its energy potential in order to contribute to the diversification of the national energy matrix.
\end{abstract}

Keywords: Biogas, Municipal solid waste, Renewable energy.

\title{
1 INTRODUÇÃO
}

O avanço da urbanização e o acesso facilitado aos bens de consumo nos países em desenvolvimento, como o Brasil, aceleraram a taxa de geração dos resíduos sólidos urbanos (RSU). Nesses países, observa-se a aplicação ineficiente de normativas, que objetivam mitigar os impactos ambientais referentes à sua disposição final, como o agravamento da polvição do solo, comprometimento das águas superficiais e subterrâneas, intensificação da polvição do ar e proliferação de vetores de doenças.

Apesar da totalidade de RSU apresentar alto percentual de matéria orgânica, os tratamentos referentes à essa parcela são incipientes no Brasil. A separação dos resíduos orgânicos e recicláveis não é uma prática difundida no território nacional. Desse modo, na melhor das hipóteses, esses são encaminhados para aterros sanitários (ALVES, 2016). Diante desse fato, para que o tratamento dos resíduos orgânicos seja eficiente é necessário a segregação dos resíduos de acordo com as características pré-definidas por cada estado ou município (IBAM, 1991). A fim de explorar as potencialidades dos resíduos orgânicos, a digestão anaeróbia pode ser utilizada para tratar esses resíduos.

O tratamento do RSU por meio da digestão anaeróbia contribui para a redução dos impactos ambientais. Ademais, esse tratamento contribui para a difusão de práticas sanitárias adequadas, como o uso correto dos aterros sanitários, implantação de sistemas de triagem eficientes, gestão abrangente dos resíduos sólidos (GOLDEMBERG; LUCON, 2007). Além de contribuir para o desenvolvimento sustentável e aumento da qualidade ambiental (BLEY JUNIOR, 2014).

Em busca da redução do volume de resíduos orgânicos dispostos nos aterros e a exploração do potencial energético desses resíduos. Esta pesquisa visa estimar o potencial de produção do biogás por meio do resíduo orgânico gerado em edifícios residenciais, de modo que essa potencialidade energética seja aproveitada para a geração de energia elétrica ou substituição do gás liquefeito de petróleo (GLP).

\section{MATERIAIS E MÉTODOS}

\subsection{Local de estudo}

A pesquisa foi realizada em edifícios residenciais situados no município de Cascavel - PR. Sendo que, os edifícios participantes deveriam conter pelo menos quatro apartamentos, menos de $10 \%$ dos apartamentos desocupados, permitir a pesagem in loco dos resíduos orgânicos acondicionados nas lixeiras e ser do mesmo padrão de acabamento. 
Assim foi escolhido quatro edifícios, esses estavam cadastrados do sistema de coleta seletiva, desse modo separavam os resíduos recicláveis dos resíduos orgânicos. De acordo com a NBR 12721:2005, os edifícios se encaixavam na classe R8-N (padrão normal).

\subsection{Pesagem da matéria orgânica}

A pesagem foi realizada durante os dias úteis da semana, exceto segunda-feira, visto que não há a coleta dos resíduos no final de semana. Desse modo, a quantidade de resíduos acondicionados na segunda-feira não corresponderia à geração diária, mas ao somatório de resíduos gerados ao longo do final de semana. Para maior abrangência do estudo, a pesagem foi realizada semanalmente ao longo do período de 11/07/2018 a 19/09/2018, assim totalizando dez pesagens.

Após as pesagens foi aplicado um checklist (Quadro 1), aos síndicos. Este contemplava perguntas acerca do número de moradores, bem como o estilo de vida desses, consumo de produtos industrializados e participação desses na separação dos resíduos orgânicos e recicláveis.

Quadro 1: Número de moradores de cada edifício

\begin{tabular}{ccccc}
\hline & Edifício A & Edifício B & Edifício C & Edifício D \\
\hline Moradores & 42 & 37 & 88 & 80 \\
\hline
\end{tabular}

O procedimento de pesagem dos resíduos orgânicos ocorreu manualmente. Para isso, utilizouse uma balança eletrônica com limite máximo de pesagem de $120 \mathrm{~kg}$. Para a obtenção da massa final, foi necessário tarar a balança, descontando o peso do tonel utilizado para armazenar o montante de sacos com os resíduos orgânicos dos moradores.

\subsection{Estimativa da capacidade de produção de biogás}

Para estimar a produção do biogás por meio da biodigestão de resíduos orgânicos, utilizouse o método de composição química de Buswell, o qual foi descrito por Chernicharo (1997):

$$
\left.\mathrm{C}_{\mathrm{n}} \mathrm{H}_{\mathrm{a}} \mathrm{O}_{\mathrm{b}} \mathrm{N}_{\mathrm{d}}+\left(\mathrm{n}-\frac{\mathrm{a}}{4}-\frac{\mathrm{b}}{2}+\frac{3 \mathrm{~d}}{4}\right)\right) \mathrm{H}_{2} \rightarrow\left(\frac{\mathrm{n}}{2}+\frac{\mathrm{a}}{8}-\frac{\mathrm{b}}{4}-\frac{3 \mathrm{~d}}{8}\right) \mathrm{CH}_{4}+\left(\frac{\mathrm{n}}{2}-\frac{\mathrm{a}}{8}+\frac{\mathrm{b}}{4}+\frac{3 \mathrm{~d}}{8}\right) \mathrm{CO}_{2}+(\mathrm{d}) \mathrm{NH}_{3}
$$

Os valores a serem utilizados na Equação 1 são descritos por Hamada (2003), referindo-se à umidade, composição química e molar dos elementos, conforme apresentado na Tabela 1:

Tabela 1 - Composição dos resíduos domiciliares.

\begin{tabular}{cccccc}
\hline Umidade (\%) & Elemento & $\begin{array}{c}\text { Porcentagem } \\
\text { em peso- } \\
\text { massa seca } \mathbf{( k g )}\end{array}$ & $\mathbf{g} / \mathbf{m o l}$ & $\begin{array}{c}\text { Total de } \\
\text { mols }\end{array}$ & $\begin{array}{c}\text { Equivalência para 1 } \\
\text { mol de nitrogênio }\end{array}$ \\
\hline \multirow{3}{*}{70} & $\mathrm{C}$ & 48 & 12,01 & 4 & 22 \\
\cline { 2 - 6 } & $\mathrm{H}$ & 6,4 & 1,01 & 6,34 & 34 \\
\hline $\mathrm{O}$ & 37,6 & 16 & 2,35 & 13 \\
\hline
\end{tabular}

Fonte: HAMADA (2003).

Posteriormente, determina-se a massa do metano e dióxido de carbono. Além de aplicar a fórmula do peso específico, conforme a Equação 2 e 3:

$V_{C H 4}=\frac{M_{C H 4}}{M_{D e s p} \times P_{C H 4}}$

Sendo:

$V_{C H 4}=$ Volume de metano $\left(\mathrm{m}^{3}\right)$; 
$M_{C H 4}=$ Massa do metano $(\mathrm{Kg})$;

$M_{\text {Desp }}=$ Massa da matéria orgânica seca $(\mathrm{Kg})$;

$P_{C H 4}=$ Peso específico do metano $\left(0,717 \mathrm{~kg} \cdot \mathrm{m}^{3}\right)$.

$V_{\mathrm{CO} 2}=\frac{M_{\mathrm{CO} 2}}{M_{\mathrm{Desp}} \times P_{\mathrm{CO} 2}}$

Sendo:

$V_{C O 2}=$ Volume do dióxido de carbono $\left(\mathrm{m}^{3}\right)$;

$M_{\mathrm{CO} 2}=$ Massa de dióxido de carbono $(\mathrm{Kg})$;

$M_{\text {Desp }}=$ Massa da matéria orgânica seca $(\mathrm{Kg})$;

$P_{\mathrm{CO}_{2}}=$ Peso específico do dióxido de carbono $\left(1,978 \mathrm{~kg} \cdot \mathrm{m}^{3}\right)$.

A quantidade de biogás produzido por $1 \mathrm{~kg}$ de resíduo orgânico (massa seca) pode ser expressa por meio da soma do resultado da Equação 2 e 3, conforme apresentado pela Equação 4:

$V_{\text {biogás }}=V_{C O 2}+V_{C H 4}$

Sendo:

$V_{\text {biogás }}=$ Volume do biogás $\left(\mathrm{m}^{3}\right)$;

$V_{C O 2}=$ Volume do dióxido de carbono $\left(\mathrm{m}^{3}\right)$;

$V_{C H 4}=$ Volume do metano $\left(\mathrm{m}^{3}\right)$

\subsection{Avaliação do potencial energético de biogás para a geração de energia elétrica e substituição do GLP}

Mediante a estimativa da produção de biogás, realizou-se a conversão dessa estimativa em energia elétrica e GLP. Para realizar a conversão utilizou-se da equivalência energética de um metro cúbico $\left(1 \mathrm{~m}^{3}\right)$ de biogás para quilowatt-hora $(\mathrm{kWh})$ e metro cúbico $\left(\mathrm{m}^{3}\right)$ de GLP, conforme a Tabela 2.

Tabela 2 - Equivalência energética de $1 \mathrm{~m}^{3}$ de biogás com energia elétrica e GLP

\begin{tabular}{cccccc}
\hline Combustíveis & $\begin{array}{c}\text { Ferraz e } \\
\text { Marriel } \\
(\mathbf{1 9 8 0})\end{array}$ & $\begin{array}{c}\text { Motta } \\
\mathbf{( 1 9 8 6 )}\end{array}$ & $\begin{array}{c}\text { Nogueira } \\
\mathbf{( 1 9 8 6 )}\end{array}$ & $\begin{array}{c}\text { Ramalho, } \\
\text { Vicchiarelli e } \\
\text { Guede (2010) }\end{array}$ & Média \\
\hline Eletricidade $(\mathrm{kWh})$ & 1,43 & 1,25 & - & 1,43 & $\mathbf{1 , 3 7}$ \\
\hline $\mathrm{GLP}\left(\mathrm{m}^{3}\right)$ & 1,45 & 1,38 & 1,43 & 1,5 & $\mathbf{1 , 4 4}$ \\
\hline
\end{tabular}

Fonte: adaptado de Oliveira (2004) e Moraes et al. (2014)

\section{RESULTADOS OBTIDOS}

\subsection{Avaliação dos Resíduos Sólidos Coletados}

Os dados obtidos da massa dos resíduos sólidos coletados se encontram na Tabela 3. 
Tabela 3 - Geração de resíduo orgânico gerado em cada dia de pesagem nos edifícios de estudo

\begin{tabular}{cccccccc}
\hline \multicolumn{2}{c}{ Edifício A } & \multicolumn{2}{c}{ Edifício B } & \multicolumn{2}{c}{ Edifício C } & \multicolumn{2}{c}{ Edifício D } \\
\hline Data & $\begin{array}{c}\text { Massa } \\
\mathbf{( k g )}\end{array}$ & Data & $\begin{array}{c}\text { Massa } \\
\mathbf{( k g )}\end{array}$ & Data & $\begin{array}{c}\text { Massa } \\
\mathbf{( k g )}\end{array}$ & Data & $\begin{array}{c}\text { Massa } \\
\mathbf{( k g )}\end{array}$ \\
\hline $11 / 07 / 2018$ & 18,9 & $11 / 07 / 2018$ & 16,4 & $12 / 07 / 2018$ & 71,5 & $12 / 07 / 2018$ & 28,8 \\
\hline $25 / 07 / 2018$ & 39,8 & $25 / 07 / 2018$ & 30,5 & $25 / 07 / 2018$ & 67,2 & $25 / 07 / 2018$ & 30,7 \\
\hline $31 / 07 / 2018$ & 19,6 & $31 / 07 / 2018$ & 15,4 & $31 / 07 / 2018$ & 54,8 & $01 / 08 / 2018$ & 31,4 \\
\hline $07 / 08 / 2018$ & 26,8 & $07 / 08 / 2018$ & 28,9 & $07 / 08 / 2018$ & 55 & $07 / 08 / 2018$ & 25,2 \\
\hline $15 / 08 / 2018$ & 30,5 & $15 / 08 / 2018$ & 29,1 & $15 / 08 / 2018$ & 58,5 & $17 / 08 / 2018$ & 30 \\
\hline $25 / 08 / 2018$ & 12,7 & $23 / 08 / 2018$ & 27,1 & $24 / 08 / 2018$ & 51,5 & $23 / 08 / 2018$ & 41,9 \\
\hline $29 / 08 / 2018$ & 34,4 & $29 / 08 / 2018$ & 21,4 & $29 / 08 / 2018$ & 52,4 & $29 / 08 / 2018$ & 35,4 \\
\hline $05 / 09 / 2018$ & 24,6 & $05 / 09 / 2018$ & 20 & $05 / 09 / 2018$ & 54,9 & $05 / 09 / 2018$ & 31,1 \\
\hline $12 / 09 / 2018$ & 16,2 & $13 / 09 / 2018$ & 19,4 & $13 / 09 / 2018$ & 53,5 & $12 / 09 / 2018$ & 32,4 \\
\hline $18 / 09 / 2018$ & 27,3 & $18 / 09 / 2018$ & 22,3 & $18 / 09 / 2018$ & 51,4 & $18 / 09 / 2018$ & 29,2 \\
\hline
\end{tabular}

Fonte: Os autores

Diante dos dados apresentados na tabela acima observa-se que a geração máxima e mínima de resíduos orgânicos no Edifício A, B, C e D foram $39,8 \mathrm{~kg}$ e 12,7 kg, 30,5 kg e 15,4 kg, $71,5 \mathrm{~kg}$ e $51,4 \mathrm{~kg}, 41,9 \mathrm{~kg}$ e $25,2 \mathrm{~kg}$, respectivamente. Observa-se, que os maiores valores de geração foram encontrados nos edifícios $C$ e $D$, visto que esses possuem maior número de moradores.

Mediante esses valores estimou-se a média diária, mensal e anual, a geração diária por morador, desvio padrão das pesagens e o coeficiente de variação, conforme Tabela 4.

Tabela 4 - Estimativas de resíduo orgânico de cada edifício estudado

\begin{tabular}{ccccc}
\hline & Edifício A & Edifício B & Edifício C & Edifício D \\
\hline Total de RSU (kg) & 250,8 & 230,5 & 570,7 & 316,1 \\
\hline Média diária (kg) & 25,08 & 23,05 & 57,07 & 31,61 \\
\hline Estimativa Mensal (kg) & 752,4 & 691,5 & $1.712,1$ & 948,3 \\
\hline Estimativa Anual (kg) & $9.154,2$ & $8.413,25$ & $20.830,55$ & $11.537,65$ \\
\hline Estimativa por morador (kg/hab/dia) & 0,597 & 0,623 & 0,648 & 0,395 \\
\hline Desvio Padrão & 8,442 & 5,491 & 6,871 & 4,756 \\
\hline Coeficiente de Variação (\%) & 33,66 & 23,82 & 12,04 & 15,05 \\
\hline
\end{tabular}

Fonte: Os autores

Diante desse cenário a geração de resíduos orgânicos foi semelhante nos edifícios A, B e C. Estes valores estão acima da geração per capita de resíduo orgânico no Brasil, visto que em 2017 a geração de RSU foi de 1,035 kg.hab dia-1 (ABRELPE, 2017). Ademais, de acordo com o IBGE (2010) 51,4\% dos RSU correspondem aos resíduos orgânicos

O edifício D apresentou divergência com a média estimada pelo IBGE (2010), uma vez que de acordo com o checklist aplicado ao síndico, o edifício fica próximo à uma faculdade de grande porte, a maioria dos moradores são universitários. Estes permanecem a maior parte do dia fora de suas residências, assim há uma menor geração de resíduos. Ademais, observase que mesmo com as oscilações feitas durante a triagem do lixo, tanto o desvio padrão como o coeficiente de variação apresentaram valores aceitáveis. 
Conforme os resultados do checklist a separação dos resíduos não é realizada de maneira correta por alguns moradores. Desse modo, evidenciou-se que alguns resíduos acondicionados nos coletores destinado aos resíduos orgânicos não se encaixavam nessa classe pois eram resíduos recicláveis, como recipientes plásticos e de papel que acondicionavam alimentos.

\subsection{Estimativa do potencial de produção do biogás}

Para estimar a produção de biogás deve-se considerar apenas a massa seca (Tabela 5). Desse modo, de acordo com Hamada (2003) cerca de $70 \%$ da massa dos resíduos orgânicos é composta por água.

Tabela 5 - Conversão da massa total para massa seca de cada edifício

\begin{tabular}{cccccccc}
\hline \multicolumn{2}{c}{ Edifício A } & \multicolumn{2}{c}{ Edifício B } & \multicolumn{2}{c}{ Edifício C } & \multicolumn{2}{c}{ Edifício D } \\
\hline $\begin{array}{c}\text { Massa } \\
\text { total (kg) }\end{array}$ & $\begin{array}{c}\text { Massa } \\
\text { seca (kg) }\end{array}$ & $\begin{array}{c}\text { Massa } \\
\text { total (kg) }\end{array}$ & $\begin{array}{c}\text { Massa } \\
\text { seca (kg) }\end{array}$ & $\begin{array}{c}\text { Massa } \\
\text { total (kg) }\end{array}$ & $\begin{array}{c}\text { Massa } \\
\text { seca (kg) }\end{array}$ & $\begin{array}{c}\text { Massa } \\
\text { total (kg) }\end{array}$ & $\begin{array}{c}\text { Massa } \\
\text { seca (kg) }\end{array}$ \\
\hline 18,9 & 5,67 & 16,4 & 4,92 & 71,5 & 21,45 & 28,8 & 8,64 \\
\hline 39,8 & 11,94 & 30,5 & 9,15 & 67,2 & 20,16 & 30,7 & 9,21 \\
\hline 19,6 & 5,88 & 15,4 & 4,62 & 54,8 & 16,44 & 31,4 & 9,42 \\
\hline 26,8 & 8,04 & 28,9 & 8,67 & 55 & 16,5 & 25,2 & 7,56 \\
\hline 30,5 & 9,15 & 29,1 & 8,73 & 58,5 & 17,55 & 30 & 9,0 \\
\hline 12,7 & 3,81 & 27,1 & 8,13 & 51,5 & 15,45 & 41,9 & 12,57 \\
\hline 34,4 & 10,32 & 21,4 & 6,42 & 52,4 & 15,72 & 35,4 & 10,62 \\
\hline 24,6 & 7,38 & 20,0 & 6,0 & 54,9 & 16,47 & 31,1 & 9,33 \\
\hline 16,2 & 4,86 & 19,4 & 5,82 & 53,5 & 16,05 & 32,4 & 9,72 \\
\hline 27,3 & 8,19 & 22,3 & 6,69 & 51,4 & 15,42 & 29,2 & 8,76 \\
\hline
\end{tabular}

Fonte: Os autores

Para a estimativa da produção de biogás utilizou-se das equações mencionadas na metodologia. Substituindo os valores apresentados na Tabela 1 na Equação 1, obteve-se:

$\mathrm{C}_{22} \mathrm{H}_{34} \mathrm{O}_{13} \mathrm{~N}_{1}+7,75 \mathrm{H}_{2} \mathrm{O} \rightarrow 11,63 \mathrm{CH}_{4}+10,38 \mathrm{CO}_{2}+\mathrm{NH}_{3}$

Posteriormente estimou-se a massa de metano e dióxido de carbono produzido na reação, assim obtendo o volume de metano e dióxido de carbono para cada quilo de resíduo orgânico seco, assim obtendo o volume de biogás produzido:

$\mathrm{V}_{\mathrm{CH} 4}=0,50 \mathrm{~m}^{3} / \mathrm{kg}$

(6)

$\mathrm{V}_{\mathrm{CO} 2}=0,44 \mathrm{~m}^{3} / \mathrm{kg}$

$V_{\text {BIOGÁS }}=0,94 \mathrm{~m}^{3} / \mathrm{kg}$

Para estimar o potencial de produção de biogás, utilizou-se os dados apresentados na Tabela 5, juntamente com os valores obtidos por meio das Equações 5 e 8, conforme apresentado na Tabela 6. 
Tabela 6 - Levantamento da geração de resíduo sólido orgânico e o potencial de produção de biogás

\begin{tabular}{cccccccc}
\hline \multicolumn{2}{c}{ Edifício A } & \multicolumn{2}{c}{ Edifício B } & \multicolumn{2}{c}{ Edifício C } & \multicolumn{2}{c}{ Edifício D } \\
\hline $\begin{array}{c}\text { Massa seca } \\
(\mathbf{k g})\end{array}$ & $\begin{array}{c}\text { Biogás } \\
\left(\mathbf{( m}^{\mathbf{3}}\right)\end{array}$ & $\begin{array}{c}\text { Massa seca } \\
(\mathbf{k g})\end{array}$ & $\begin{array}{c}\text { Biogás } \\
\left(\mathbf{m}^{3}\right)\end{array}$ & $\begin{array}{c}\text { Massa seca } \\
(\mathbf{k g})\end{array}$ & $\begin{array}{c}\text { Biogás } \\
\left(\mathbf{m}^{3}\right)\end{array}$ & $\begin{array}{c}\text { Massa seca } \\
(\mathbf{k g})\end{array}$ & $\begin{array}{c}\text { Biogás } \\
\left(\mathbf{m}^{3}\right)\end{array}$ \\
\hline 5,67 & 5,33 & 4,92 & 4,62 & 21,45 & 20,16 & 8,64 & 8,12 \\
\hline 11,94 & 11,22 & 9,15 & 8,60 & 20,16 & 18,95 & 9,21 & 8,66 \\
\hline 5,88 & 5,53 & 4,62 & 4,34 & 16,44 & 15,45 & 9,42 & 8,85 \\
\hline 8,04 & 7,56 & 8,67 & 8,15 & 16,5 & 15,51 & 7,56 & 7,11 \\
\hline 9,15 & 8,60 & 8,73 & 8,21 & 17,55 & 16,50 & 9,0 & 8,46 \\
\hline 3,81 & 3,58 & 8,13 & 7,64 & 15,45 & 14,52 & 12,57 & 11,82 \\
\hline 10,32 & 9,70 & 6,42 & 6,03 & 15,72 & 14,78 & 10,62 & 9,98 \\
\hline 7,38 & 6,94 & 6,0 & 5,64 & 16,47 & 15,48 & 9,33 & 8,77 \\
\hline 4,86 & 4,57 & 5,82 & 5,47 & 16,05 & 15,09 & 9,72 & 9,14 \\
\hline 8,19 & 7,70 & 6,69 & 6,29 & 15,42 & 14,49 & 8,76 & 8,23 \\
\hline
\end{tabular}

Fonte: Os autores

Por meio das estimativas de produção de biogás nota-se que a geração máxima e mínima de biogás no Edifício A, B, C e D seriam de aproximadamente $11,2 \mathrm{~m}^{3}$ e $3,5 \mathrm{~m}^{3}, 8,6 \mathrm{~m}^{3}$ e 4,3 $\mathrm{m}^{3}$, $20,1 \mathrm{~m}^{3}$ e $14,4 \mathrm{~m}^{3}, 11,8 \mathrm{~m}^{3}$ e $7,1 \mathrm{~m}^{3}$, respectivamente.

\subsection{Conversão do biogás em eletricidade e GLP}

Mediante a estimativa da geração de biogás, juntamente com a média do fator de conversão para GLP e eletricidade, foi possível realizar a equivalência conforme se apresenta na Tabela 7.

Tabela 7 - Equivalência do biogás com energia elétrica e GLP

\begin{tabular}{|c|c|c|c|c|c|}
\hline & & Total & Média Diária & Estimativa Mensal & Estimativa Anual \\
\hline \multirow{4}{*}{ Edifício A } & Massa seca $(\mathrm{kg})$ & 75,240 & 7,524 & 225,720 & $2.746,260$ \\
\hline & Biogás $\left(\mathrm{m}^{3}\right)$ & 70,726 & 7,073 & 212,177 & $2.581,484$ \\
\hline & Energia Elétrica (kWh) & 96,89407 & 9,689 & 290,682 & $3.536,634$ \\
\hline & $\operatorname{GLP}\left(\mathrm{m}^{3}\right)$ & 101,8449 & 10,184 & 305,535 & $3.717,338$ \\
\hline \multirow{4}{*}{ Edifício B } & Massa seca $(\mathrm{kg})$ & 69,150 & 6,915 & 207,450 & $2.523,975$ \\
\hline & Biogás $\left(\mathrm{m}^{3}\right)$ & 65,001 & 6,500 & 195,003 & $2.372,537$ \\
\hline & Energia Elétrica (kWh) & 89,05137 & 8,905 & 267,154 & $3.250,375$ \\
\hline & $\operatorname{GLP}\left(\mathrm{m}^{3}\right)$ & 93,60144 & 9,360 & 280,804 & $3.416,453$ \\
\hline \multirow{4}{*}{ Edifício C } & Massa seca $(\mathrm{kg})$ & 171,210 & 17,121 & 513,630 & $6.249,165$ \\
\hline & Biogás $\left(\mathrm{m}^{3}\right)$ & 160,937 & 16,094 & 482,812 & $5.874,215$ \\
\hline & Energia Elétrica (kWh) & 220,4842 & 22,048 & 661,453 & $8.047,675$ \\
\hline & $\operatorname{GLP}\left(\mathrm{m}^{3}\right)$ & 231,7499 & 23,175 & 695,250 & $8.458,870$ \\
\hline \multirow{4}{*}{ Edifício D } & Massa seca (kg) & 94,830 & 9,483 & 284,490 & $3.461,295$ \\
\hline & Biogás $\left(\mathrm{m}^{3}\right)$ & 89,140 & 8,914 & 267,421 & $3.253,617$ \\
\hline & Energia Elétrica (kWh) & 122,1221 & 12,212 & 366,366 & $4.457,456$ \\
\hline & $\operatorname{GLP}\left(\mathrm{m}^{3}\right)$ & 128,3619 & 12,836 & 385,086 & $4.685,209$ \\
\hline
\end{tabular}

Fonte: Os autores 
Mediante os valores apresentados na tabela acima, observa-se que os maiores valores para a produção de biogás encontraram-se nos edifícios $C$ e $D, 5.874,215 \mathrm{~m}^{3}$ e $3.253,617 \mathrm{~m}^{3}$, respectivamente. Desse modo, por meio das produções anuais de biogás, estima-se que o edifício C obteria $8046,675 \mathrm{kWh}$ de energia elétrica ou 8458,87 $\mathrm{m}^{3}$ de GLP. Enquanto o Edifício D obteria 4457,456 kWh de energia elétrica ou 4685,209 $\mathrm{m}^{3}$ de GLP.

\section{CONCLUSÃO}

Desse modo conclui-se que a produção diária de resíduos orgânicos por morador nos edifícios abordados é próxima a geração nacional apresentada na bibliografia. Já o edifício D apresentou sua geração de resíduos orgânicos menor que a média nacional. Essa perspectiva é reflexo do modo de vida de muitos moradores deste edifício, uma vez que esses realizam a maioria das refeições em outros lugares, assim por consequência geram menos resíduos orgânicos.

Por meio da análise da geração de resíduos orgânicos dos quatro edifícios estudados, concluise que há um descarte expressivo de matéria orgânica. Os edifícios A, B, C e D apresentaram os seguintes valores de geração de resíduos orgânicos, respectivamente: 0,597 kg.hab.dia-1, $0,623 \mathrm{~kg} \cdot \mathrm{hab} \mathrm{dia}^{-1}, 0,648 \mathrm{~kg} \cdot \mathrm{hab} \mathrm{dia}^{-1}$ e 0,395 kg.hab dia ${ }^{-1}$. Com relação ao fator de conversão do biogás, produto da digestão anaeróbia dos resíduos orgânicos, os edifícios $A, B, C$ e D poderiam produzir anualmente $2.581,484 \mathrm{~m}^{3}, 2.372 .537 \mathrm{~m}^{3}, 5.874,215 \mathrm{~m}^{3}$ e $3.253,617 \mathrm{~m}^{3}$, respectivamente.

Diante desse cenário se a parcela de resíduos orgânicos gerados nos edifícios fosse tratada por meio da digestão anaeróbia seria possível explorar o potencial energético do biogás e utilizá-lo como fonte alternativa de energia. Desse modo, é necessário a coleta segregada dos resíduos, a fim de aumentar a fração de resíduos tratados e diminuir o volume de resíduos aterrados.

\section{REFERÊNCIAS}

ALVES, I. R. de F. S. Avaliação da codigestão na produção de biogás. 2016. 168 p. Tese (Doutorado em Engenharia Civil) - Universidade Federal do Rio de Janeiro. Rio de Janeiro, 2016.

ASSOCIAÇÃO BRASILEIRA DE EMPRESAS DE LIMPEZA PÚBLICA DE RESÍDUOS ESPECIAIS - ABRELPE. Panorama dos resíduos sólidos no Brasil 2017. São Paulo, 2017.

ASSOCIAÇÃO BRASILEIRA DE NORMAS TÉCNICAS - ABNT. NBR 12721: Avaliação de custos de construção para incorporação imobiliária e outras disposições para condomínios edilícios. Rio de Janeiro, 2005.

BARCELOS, B. R. Avaliação de diferentes inóculos na digestão anaeróbia da fração orgânica de resíduos sólidos domésticos. Dissertação (Mestrado em Tecnologia Ambiental e Recursos Hídricos) - Universidade de Brasília, Brasília, 2009. 90 p.

BLEY JUNIOR, C. Biogás - A energia invisível. 2a ed. São Paulo: Cibiogás, 2014. 231 p.

BRASIL. Lei n. 12.305, de 2 de agosto de 2010. Institui a Política Nacional de Resíduos Sólidos. Diário Oficial da República Federativa do Brasil, Poder Executivo, Brasília-DF, 2 ago. 2010.

CHERNICHARO, C. A. L. Reatores anaeróbicos. Belo Horizonte: DESA, 1997.

DA SILVEIRA, L. A.; BERTÉ, R.; PELANDA, M. A. Gestão de Resíduos Sólidos - cenários e mudanças de paradigmas. $1^{a}$ ed. Editora Intersaberes: Curitiba, 2018. 226 p. 
GOLDEBERG, J.; LUCON, O. Energias renováveis: um futuro sustentável. Revista USP, n. 72, p. 615, 2007.

HAMADA, J. Resíduos Sólidos: Conceitos e caracterização. Bauru: GERESOL, 2003.

INSTITUTO BRASILEIRO DE ADMINISTRAÇÃO MUNICIPAL - IBAM. Cartilha de Limpeza Urbana. Rio de Janeiro, $1991.81 \mathrm{p}$.

MORAES JUNIOR, L. T. R. Recuperação energética de resíduos no médio vale do rio Itajaí AçU com foco na conversão em energia elétrica. Dissertação (Mestrado em Engenharia Elétrica) Universidade Regional de Blumenau. Blumenau, 2012.

MORAES, M. L.; BACCHI, M. R. P. Ełanol: do início às fases atuais de produção. Revista de Política Agrícola, v. 23, n. 4, p. 5-22, 2014.

OLIVEIRA, S. V. W. B. Modelo para tomada de decisão na escolha do sistema de tratamento de esgoto. Tese (Doutorado em Administração) - Universidade de São Paulo, São Paulo, 2004. 